\title{
Interaction of the full-length heme-based CO sensor protein RcoM-2 with
}

\section{ligands}

\author{
Mayla Salman\#, Carolina Villamil Franco ${ }^{\#, \dagger}$, Rivo Ramodiharilafy, Ursula Liebl \\ \& Marten H. Vos*
}

LOB, CNRS, INSERM, Ecole Polytechnique, Institut Polytechnique de Paris, 91128 Palaiseau Cedex, France

*Corresponding author, marten.vos@polytechnique.edu, tel. +33169335066

${ }^{\dagger}$ Present adress : LIDYL, CEA, CNRS, Université Paris Saclay, 91191 Gif-sur-Yvette, France

${ }^{\#}$ M.S. and C.V.F. contributed equally to this work 


\section{ABSTRACT}

The heme-based and CO-responsive RcoM transcriptional regulators from Burkholderia xenovorans are known to display an extremely high affinity for CO while being insensitive to $\mathrm{O}_{2}$. We have quantitatively characterized the heme-CO interaction in full-length RcoM-2 and compared it with the isolated heme domain RcoMH-2 to establish the origin of these characteristics. Whereas the CO binding rates are similar to those of other heme-based sensor proteins, the dissociation rates are two to three orders of magnitude lower. The latter property is tuned by the yield of CO escape from the heme pocket after disruption of the hemeCO bond, as determined by ultrafast spectroscopy. For the full-length protein this yield is $\sim 0.5 \%$ and for the isolated heme domain even lower, associated with correspondingly faster $\mathrm{CO}$ rebinding kinetics, leading to $K_{d}$ values of 4 and $0.25 \mathrm{nM}$, respectively. These differences imply that the presence of the DNA-binding domain influences the ligand-binding properties of the heme domain, thus abolishing the observed quasi-irreversibility of CO binding to the isolated heme domain. RcoM-2 binds target DNA with high affinity $\left(\mathrm{K}_{d}<2 \mathrm{nM}\right)$ when CO is bound to the heme, and the presence of DNA also influences the heme-CO rebinding kinetics. The functional implications of our findings are discussed.

For Table of Contents use only

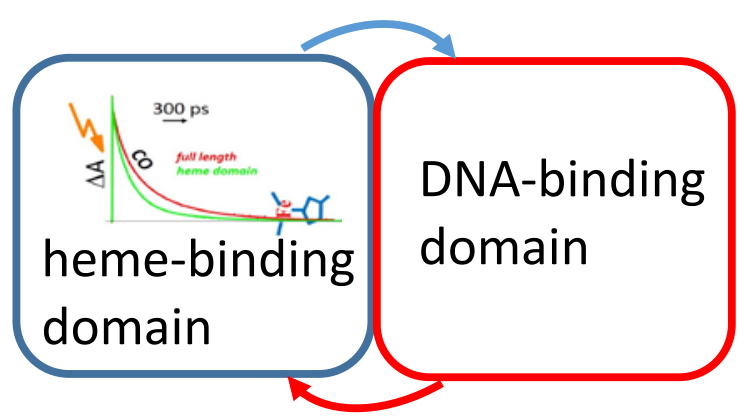




\section{INTRODUCTION}

An important subset of the large family of heme proteins is constituted by heme-based gas sensor proteins $(1,2)$. These proteins play crucial roles in the initial adaptation processes of organisms to changing environmental gas conditions usually by altering the expression of specific genes upon binding or release of specific small molecules to protein-bound heme. Sensors of the highly reactive signaling and respiratory molecules $\mathrm{NO}$ and $\mathrm{O}_{2}$ have obviously gained considerable attention. More recently, the more inert CO molecule was also established as an important sensor molecule (3-6). Atmospheric CO is generated by natural and industrial processes and as a eukaryotic heme breakdown product, and many prokaryotes possess distinct CO-regulated systems that catalyze CO-oxidation. In mammalian systems, the transcription factor NPAS2 (7) and the SUR2A receptor-associated $\mathrm{K}_{\text {ATP }}$ channel (8) have been reported to be CO-responsive (with CO affinity around $1 \mu \mathrm{M}$ ), but the physiological relevance of these responses remains debated. On the other hand, to date two distinct prokaryotic CO-specific sensor proteins, CooA and RcoM, have been described; both are single-component transcriptional regulators that are activated upon binding of $\mathrm{CO}$ to an associated heme domain. CooA, involved in the regulation of anaerobic CO metabolism in Rhodospirillum rubrum, is relatively well-studied, and crystallographic models are available $(9,10)$. RcoM (regulator of CO metabolism) proteins have been discovered a decade ago and are active in the aerobic bacterium Burkholderia xenovorans (11). They are less well characterized (in particular structural data are lacking), but display some surprising properties (11-16).

B. xenovorans expresses two RcoM proteins, RcoM-1 and RcoM-2, which are highly homologous (11). Each is comprised of an N-terminal PAS heme-binding sensor domain and a C-terminal LytTR DNA-binding effector domain. RcoM-1 has been reported to form homodimers (17). In the absence of external ligands, in both the ferric and the ferrous state the heme is six-coordinate with intrinsic amino acids as axial ligands. In its ferrous state, it can bind 
$\mathrm{CO}$ and NO while forming 6-coordinate complex with a proximal histidine (15). In the ferrous complex, binding of an external ligand is thought to displace a distal methionine residue (13, 15), triggering a conformational change that activates the C-terminal DNA-binding domain. In particular, for RcoM-1 it has been demonstrated that target DNA binds in the CO-bound state, but not in the ferric state and the ferrous state in the absence of $\mathrm{CO}$ (14). The $\mathrm{O}_{2}$-complex appears not to be stably formed, which presumably allows the protein to act as a CO sensor under aerobic conditions. Other than for CooA and the mammalian CO-responsive systems, the CO affinity is extremely high, at least in the low nanomolar regime for full-length RcoM-1 (14) and even sub-nanomolar for the isolated heme sensor domain of RcoM-2 (12). Indeed, the proteins are purified with a high amount of ferrous CO-complex present after standard heterologous expression in Escherichia coli. The bound CO can be permanently removed from the heme only by prolonged illumination under aerobic conditions $(11,12)$. Intrigued by these findings, we have previously studied the kinetic properties of CO binding to the RcoM-2 heme domain (termed RcoMH-2). These revealed unique kinetics properties including a quasi-unity (>99\%) yield of heme-CO geminate rebinding on the picosecond timescale upon photocleavage of the heme-CO bond (12). This indicates that CO can hardly escape from the heme pocket and makes the RcoMH-2 construct a promising oxygen-compatible $\mathrm{CO}$ scavenger. On the other hand, the question arises how RcoM-2 can physiologically function as a true sensor of the CO concentration if CO-binding appears quasi-irreversible. Here, this question is investigated by performing ligand-binding studies on the full-length RcoM-2 protein and also by determining the effects of RcoM binding to DNA. We show substantially different heme-ligand interactions in the heme sensor domain in the presence of the DNA-binding domain that lead to more reversible binding of $\mathrm{CO}$. 


\section{MATERIALS AND METHODS}

The full-length RcoM-2 protein (amino acids 1-267, UniprotKB Q13IY4) was heterologously expressed in E. coli BL21 DE3 from a pQE80L expression vector (QIAGEN) using $1 \mathrm{mM}$ isopropyl- $\beta$-D-thiogalactopyranoside. RcoM-2 (expected MW 30.5 kDa), containing the N-terminal heme-containing PAS domain (amino acids 15-86) and the Cterminal HTH LytTR-type DNA-binding response domain (amino acids 161-266) was purified by affinity chromatography on Ni-TED columns (Protino Ni-TED, Macherey Nagel) and eluted with $50 \mathrm{mM} \mathrm{NaH}_{2} \mathrm{PO}_{4}, 300 \mathrm{mM} \mathrm{NaCl}$ and $250 \mathrm{mM}$ imidazole, $\mathrm{pH}$ 8.0, followed by imidazole removal on Econo-Pac columns (Bio-Rad). The heme-containing PAS sensor domain RcoMH-2 was expressed and purified as described (12). The purified proteins were suspended in $50 \mathrm{mM}$ Tris-HCl buffer, $\mathrm{pH}$ 8.0, $150 \mathrm{mM} \mathrm{NaCl}$ and, unless otherwise indicated, all experiments were performed in this buffer.

The heme-containing PAS sensor domain RcoMH-2 was purified almost entirely in the ferrous CO-bound form, whereas this was only the case for a small fraction of full-length RcoM-2. For experiments on other forms than the CO-bound form, the samples were first transformed fully to the ferric form by illumination for $\sim 12 \mathrm{~h}$ with white light originating from a halogen lamp equipped with an optical fiber at an intensity of $\sim 0.15 \mathrm{~W} / \mathrm{cm}^{2}$, at $4^{\circ} \mathrm{C}$ in an airexposed vial. To obtain the ferrous unliganded form, the ferric protein was degassed using a gas train and reduced with a slight excess of dithionite. The ferrous NO form was fully formed upon exposure of the ferrous protein to 0.1 atm NO in the cell headspace. To obtain the fully CO-bound form, as-purified protein was reduced with a slight excess of dithionite and exposed to $1 \mathrm{~atm} \mathrm{CO}$ in the cell headspace.

All steady state and ultrafast spectroscopy experiments were performed in 1-mm cells that could be sealed gastight with rubber stoppers. Steady-state spectra were obtained using a Shimadzu UV-Vis 1700 spectrometer. Ultrafast transient absorption experiments at a $500 \mathrm{~Hz}$ 
repetition rate, with a $570 \mathrm{~nm}$ pump pulse and a continuum probe pulse, were performed as in ref. (18) using a heme concentration of $\sim 30 \mu \mathrm{M}$. To reduce baseline fluctuation noise the data are plotted as difference kinetics between the induced maximum and the bleaching minimum in the Soret absorption regions. To correct for beam-overlap, drifts on the nanosecond timescale kinetics after photodissociation of CO-myoglobin (where no rebinding occurs on the investigated timescale) were used as reference.

Stopped-flow experiments to monitor CO binding to the ferrous protein were performed in a Biologic SFM 300 instrument equipped with a JM Tidas diode detector. The optical path length of the measuring cell was $0.8 \mathrm{~mm}$. The instrument was extensively flushed with gaseous nitrogen prior to use. After mixing, the protein concentration was $\sim 25 \mu \mathrm{M}$.

The rate of CO dissociation was determined by the NO replacement method (19). Here, starting from the fully CO-bound form, CO in the cell head space was replaced by 1 atm NO. The sample was then kept in the dark and spectra were taken at regular intervals.

Fluorescence anisotropy-based DNA-binding assays with full-length RcoM-2 were performed using 5' Texas-Red fluorophore-labeled DNA in 1x1 cm optical path length cells. Purified and CO-dissociated protein was suspended in $50 \mathrm{mM}$ Tris-HCl, pH 7.6 and $100 \mathrm{mM}$ $\mathrm{NaCl}$ at a concentration of $1 \mu \mathrm{M}$. The ferrous and CO-bound forms were prepared in gastight $1 \times 1 \mathrm{~cm}$ optical path length cells as described above.

An 18-mer of double stranded DNA, comprising the equivalent of region a of the RcoM-2 DNA binding region (14), was obtained by mixing equimolar amounts of the two complementary oligonucleotides 5'-TCGATTTTCGCGCAAATT-3' (forward) and 5'AATTTGCGCGAAAATCGA-3' (reverse) (Eurogentec). The 5'-extremity of the forward oligonucleotide was labeled with Texas Red. The oligonucleotides were heated three minutes at $80^{\circ} \mathrm{C}$ followed by slow cooling down to room temperature to allow complete annealing. The DNA concentration in the assays was $6.4 \mathrm{nM}$. For the experiments with reduced protein, the 
DNA stock solution was degassed in the sealed optical cells and $\sim 0.5 \mathrm{mM}$ dithionite was added. For the experiments with CO-liganded protein, it was in addition equilibrated with $1 \mathrm{~atm} \mathrm{CO}$ atmosphere. RcoM-2 in the different forms was added stepwise using a gastight Hamilton syringe up to concentrations of $25 \mathrm{nM}$. Fluorescence anisotropy was measured following a procedure as described (20) in a Cary Eclipse fluorometer equipped with a manual polarization device, with an excitation beam centered at $590 \mathrm{~nm}$ and by scanning the Texas Red emission spectrum. Anisotropy data were fitted to a tight binding formalism $(21,22)$ to estimate the binding constant $\left(K_{d}\right)$.

All experiments were performed at room temperature.

\section{RESULTS}

Steady-state absorption spectra. Fig. 1 shows the steady-state absorption spectra of RcoM-2 under different conditions. The as-prepared, reduced, and carbonylated spectra are similar to those reported previously by Marvin et al. (15) that were assigned to FeIII His-Cys, FeII His-Met and FeII His-CO coordinated heme-complexes, respectively. Yet we note that upon prolonged illumination, the spectrum changes somewhat, with a modestly blue-shifted and broadened Soret band and a less pronounced shoulder around $570 \mathrm{~nm}$. This finding indicates that a fraction of $\mathrm{CO}$ was present in the as-purified protein. This fraction is far smaller than in the isolated heme domain RcoMH-2 that is almost completely purified in the CO-bound form (12). For RcoM-1, differences in CO-binding in the purified protein have been related to differences in protein expression (14). We used the same expression system and E. coli growth conditions for our full-length and heme domain constructs. Therefore we assign this difference to intrinsic differences in $\mathrm{CO}$ affinity, as will be quantified below. 


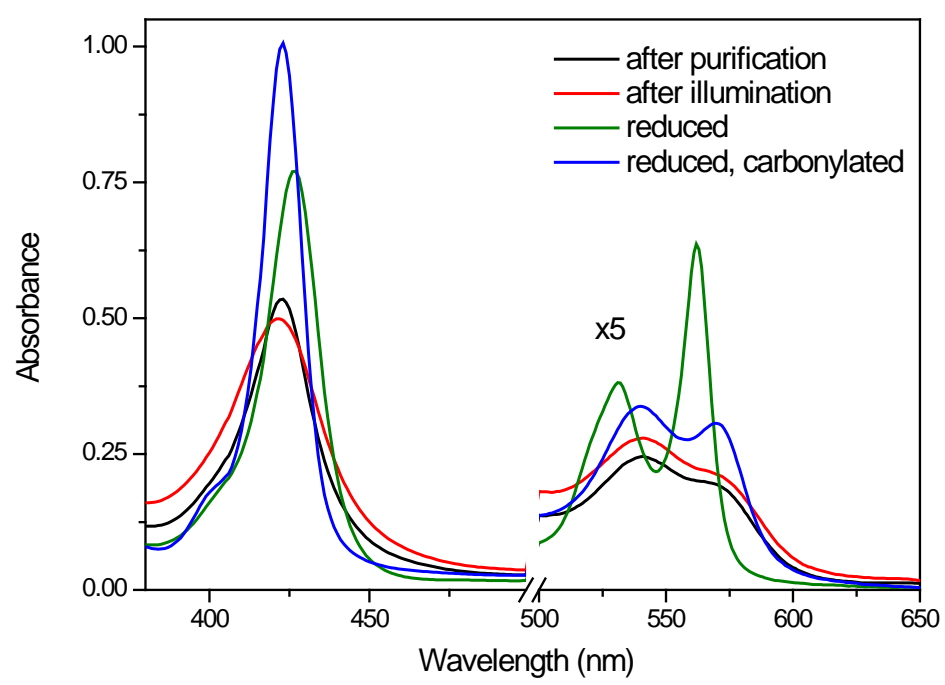

Figure 1. Absorption spectra of RcoM-2, subsequently after purification, after prolonged illumination, after degassing and reduction with sodium dithionite, and after exposure to $1 \mathrm{~atm}$. CO.

The spectra of the fully reduced and CO-bound form are very similar in the full-length protein and the purified heme domain, although small $(\sim 1 \mathrm{~nm})$ shifts are observed (Table S1). By contrast, the FeIII (as-prepared illuminated) form is substantially blue-shifted in the RcoMH-2 construct; this can be assigned to the presence of a fraction of 5-coordinate complex (12).

Bimolecular CO binding. CO binding to RcoM-2 was monitored by stopped-flow spectroscopy. Fig. 2A shows spectra at different delay times after mixing reduced RcoM-2 with CO. The initial spectrum corresponds to the 6-coordinate His-Met complex and the final one to the 6-coordinate His-CO complex (cf. Fig. 1). The spectra show clear isosbestic points and no indication for the population of distinct intermediate species. The kinetics can be well fit with single-exponential kinetics (Fig. 2B) that vary linearly with CO concentration (Fig. 2B, inset) yielding an apparent bimolecular rate constant $k_{o b s}$ of $16 \times 10^{3} \mathrm{M}^{-1} \mathrm{~s}^{-1}$. In principle, for such a ligand exchange reaction the rate is described by a hyperbolic function (cf. Refs. (12, 23))

$$
k_{o b s}=\frac{k_{-M} k_{C O}[\mathrm{CO}]}{k_{M}+k_{C O}[\mathrm{CO}]}
$$


where $k_{-M}$ is the rate of dissociation and displacement of the intrinsic (methionine) ligand, $k_{M}$ the rate of association of the intrinsic ligand $\left(K_{M}=k_{M} / k_{-M}>>1\right.$, as the protein is initially predominantly 6-coordinate), and $k_{C O}$ is the bimolecular rate constant of CO binding to the 5coordinate heme. Within the tested [CO] range, no rate saturation was observed, so the rate is not limited by dissociation of the intrinsic methionine, and $k_{-M}>>5 \mathrm{~s}^{-1} \cdot k_{o b s}$ then corresponds to $k_{C O} / K_{M}$.

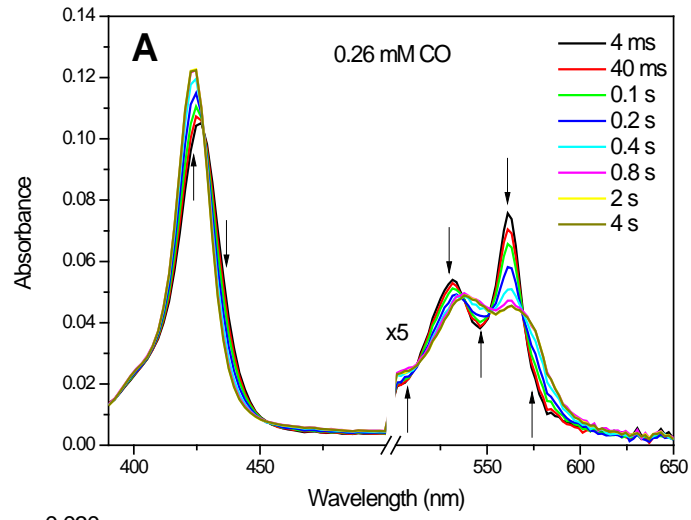

Figure 2. Stopped-flow spectroscopy of CO binding to RcoM-2. A. Spectra at different delay times after mixing to a final $\mathrm{CO}$ concentration of $0.26 \mathrm{mM}$. B. Kinetics at $420 \mathrm{~nm}$ at various

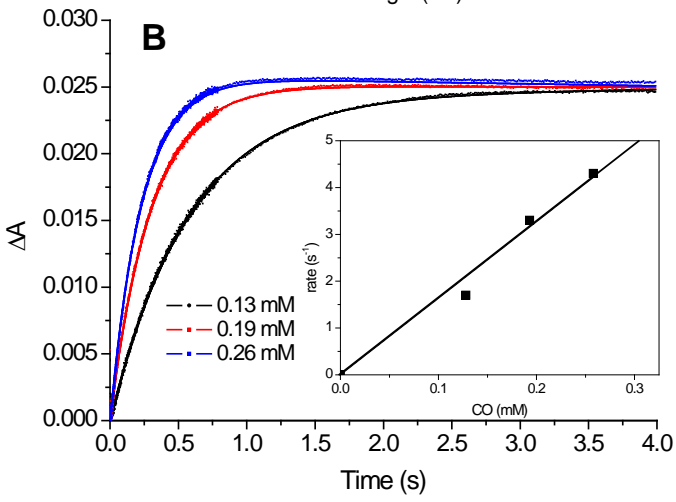
CO concentrations. Inset: Linear fit to rates corresponding to $k_{o b s}=16 \mathrm{x}$ $10^{3} \mathrm{M}^{-1} \mathrm{~s}^{-1}$.

These findings are distinct from those that we reported earlier for the purified heme domain RcoMH-2, but also have some common properties: in RcoMH-2 the kinetics were biphasic, with the main phase displaying a very similar value for $k_{C O} / K_{M}\left(15 \times 10^{3} \mathrm{M}^{-1} \mathrm{~s}^{-1}\right)$, but clear saturation behaviour corresponding to $k_{-M}=5 \mathrm{~s}^{-1}$. Thus motion of the intrinsic distal heme ligand appears less restricted in the full-length protein. 
CO dissociation. The rate of thermal CO dissociation from the protein was determined by exposing the CO-bound protein to excess $\mathrm{NO}$ and monitoring the spectral changes associated with replacement of CO by NO as a function of time (Fig. 3A). To avoid light-induced CO dissociation, the samples were kept in the dark between monitoring subsequent spectra. The kinetics (Fig. 3B) are plotted as peak-to-bleaching absorption difference to minimize the effect of baseline variations. They are well-described by single-exponential kinetics with a time constant of $4.4 \mathrm{~h}$, corresponding to a CO off rate of $64 \times 10^{-6} \mathrm{~s}^{-1}$.

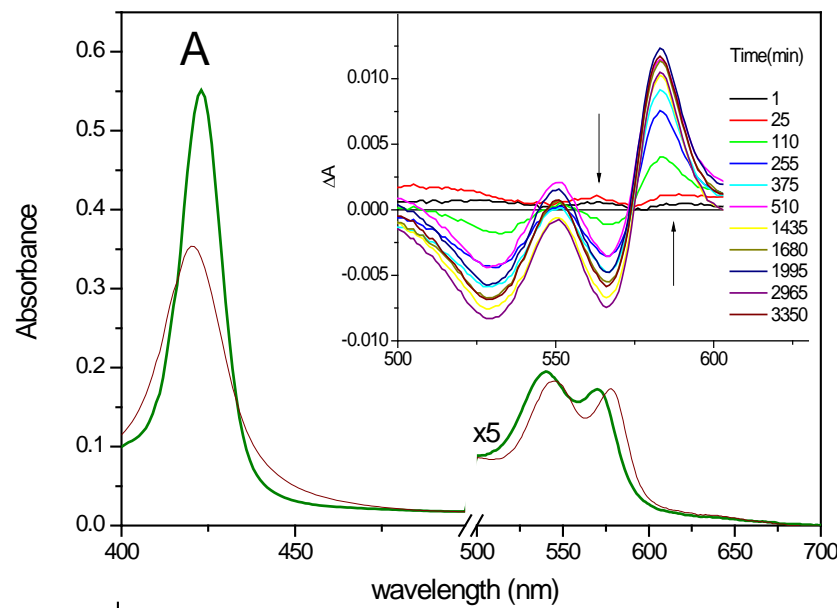

Figure 3. $\mathrm{CO}$ dissociation kinetics. A. RcoM-2 spectra of the CO-bound form (green) and after equilibration of this form with NO for $56 \mathrm{~h}$ (red). Inset: difference spectra in the $\mathrm{Q}$ band region at different delay times. B. Kinetics of CO replacement by NO for fulllength RcoM-2 (black) and the heme domain RcoMH-2 (red). Lines are single exponential fits. Note the change in time scale.

This time constant is much shorter than the rough estimation in the order of days that we previously advanced for the heme domain RcoMH-2 (12). We therefore used the same method to precisely determine the time constant for RcoMH-2 at $80 \mathrm{~h}$ (CO off rate $3.5 \times 10^{-6} \mathrm{~s}^{-1}$ ) and found it indeed to be almost 20-fold longer than for the full-length protein. 
Heme-CO geminate rebinding. Recombination upon photodissociation of the hemeCO bond was investigated using ultrafast pump-probe spectroscopy. The kinetics (Fig. 4, red curve) are characterized by a phase of a few picoseconds that reflects mostly photophysical processes (24) and multiphasic rebinding that can be fitted (Table 1) with time constants of 170 ps and 660 ps (with 45/55 amplitude ratio). The rebinding is substantially slower than in the isolated heme domain (green curve, fit constants 170 ps and 500 ps, 65/35 amplitude ratio (12)). Moreover, for the full-length protein a small but significant asymptotic value of $0.5 \%$ was found, which reflects CO escape from the heme pocket, whereas in the heme domain rebinding was complete within experimental error.

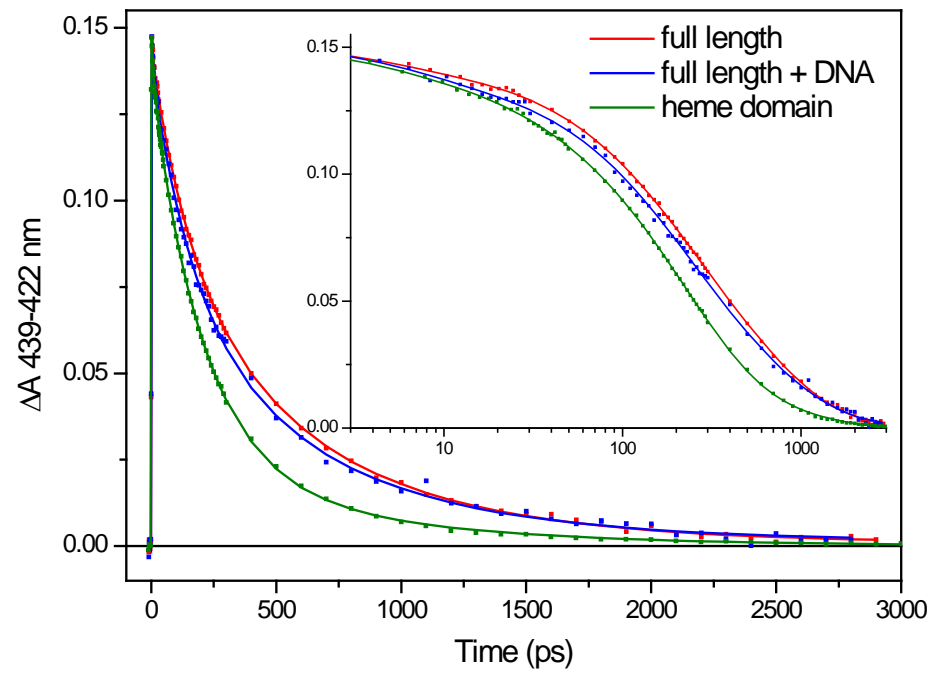

Figure 4. Rebinding kinetics of CO to RcoM-2 after photodissociation in the absence and presence of DNA. The corresponding kinetics for RcoMH-2 (12) are also given. Inset: same on a logarithmic time scale

Table 1. Fit parameters for the kinetics of heme-CO recombination (Fig. 4). The data on the time scale $>10$ ps were fitted to a function of the form $A_{1} e^{-t / \tau_{1}}+A_{2} e^{-t / \tau_{2}}+A_{3}$. The amplitudes are normalised to 1 and the time constants are in picoseconds.

\begin{tabular}{l|lllll} 
& $\tau_{1}$ & $\mathrm{~A}_{1}$ & $\tau_{2}$ & $\mathrm{~A}_{2}$ & $\mathrm{~A}_{3}$ \\
\hline RcoMH-2 & 170 & 0.35 & 500 & 0.65 & 0.00 \\
RcoM-2 & 180 & 0.453 & 660 & 0.541 & 0.006 \\
RcoM-2 + DNA & 170 & 0.514 & 710 & 0.482 & 0.004
\end{tabular}


Heme-NO geminate rebinding. NO rebinding to the full-length protein was also investigated (Fig. 6). Faster kinetics as with $\mathrm{CO}$ are observed, as is the case in most heme proteins (24). The kinetics can be fit by two phases of 8 ps and 300 ps (12\%) which are substantially slower and more dispersed than those of the corresponding kinetics of the heme domain (6 ps and 130 ps (5\%) (12)). The trend is similar as with CO recombination and in full agreement with more conformational freedom for the dissociated ligand in the full-length protein.

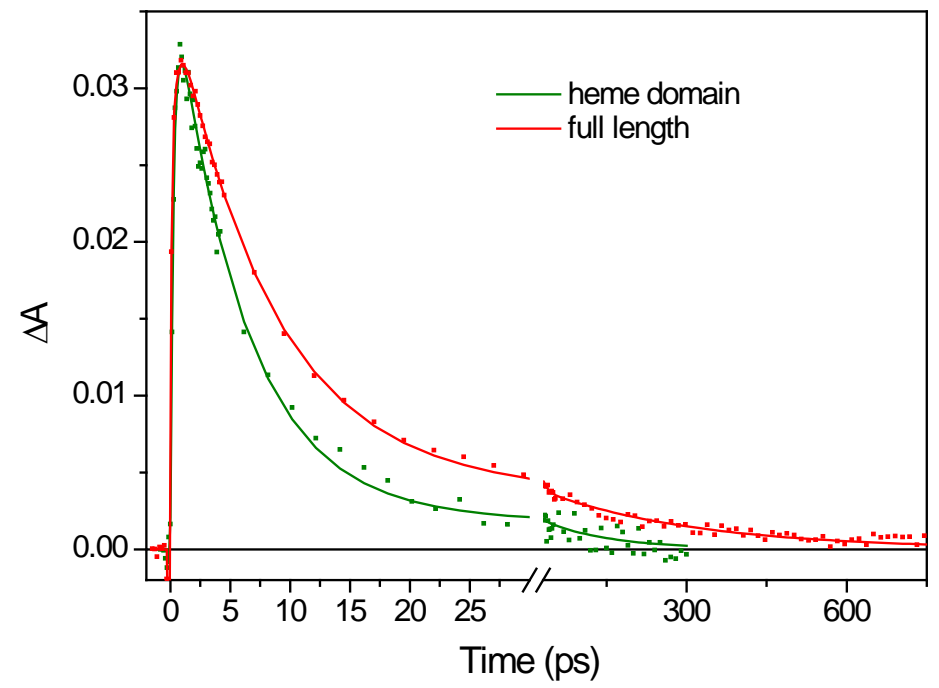

Figure 5. Rebinding kinetics of NO to ferrous RcoM-2 after photodissociation. The corresponding kinetics for RcoMH-2 (ref. 12) is also given.

DNA binding. Previous work using fluorescence anisotropy indicated that RcoM-1 binds DNA only in the CO-bound form, but the affinity could not be determined (14). Here, measuring fluorescence anisotropy of a 5'-Texas Red-labelled target DNA sequence as a function of RcoM-2 protein concentration (Fig. 6), we show that the affinity for the CO-bound form of this protein is very high $\left(\mathrm{K}_{\mathrm{d}}<2 \mathrm{nM}\right)$ and that the FeIII form of the protein does not bind the target DNA. 


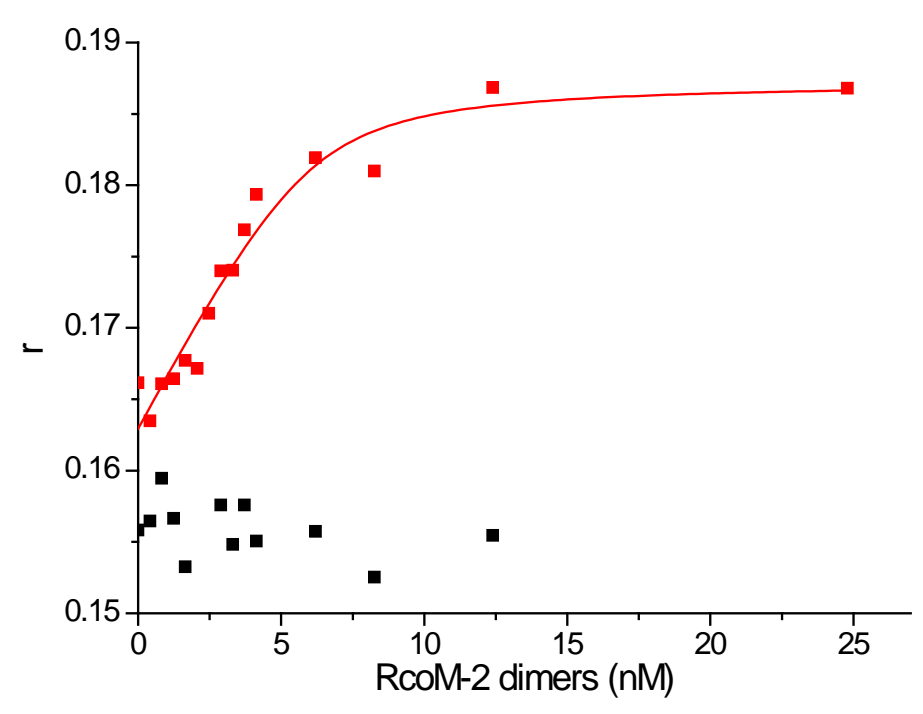

Figure 6. Fluorescence anisotropy of 5' Texas Redlabelled target DNA as a function of RcoM-2 concentration in the FeIII state (black) and CO-bound state (red). The fit is to a tightbinding formalism for RcoM2 dimers and indicates that CO-bound RcoM-2 binds DNA stoichiometrically with a $K_{d}<2$ nM.

Effect of DNA binding on CO-bound protein. Adding unlabelled target DNA to the CO-bound RcoM-2 protein did not have any measurable effect on the shape of the absorption spectrum (Table S1). However, a small but significant effect was observed on the rebinding of CO (Fig. 4, blue curve, Table 1) that overall accelerated: the fit constants were similar to those in the absence of DNA, but the amplitude ratio changed to $\sim 50 / 50$. The fractional escape was found to be unchanged within experimental error. The effect on the decay is in the same direction as that observed for DNA binding to the CooA CO sensor (25). As discussed in detail in the Discussion section, this result suggests that DNA binding somewhat favors more rigid conformations of the heme domain.

\section{DISCUSSION}

We have investigated the heme-ligand (in particular heme-CO) interactions of fulllength RcoM-2 and compared them with those of the isolated heme domain RcoMH-2. In general terms, the binding properties were found to be quite different, with RcoM-2 displaying weaker binding of CO than RcoMH-2. A 20-fold higher CO dissociation rate is observed in 
RcoM-2. This feature can be related to the fact that despite the extremely high CO recombination yield a sizeable fraction $(\sim 0.5 \%)$ of photodissociated CO escapes the heme pocket in the full-length protein RcoM-2, but not in RcoMH-2. Thus in the presence of the DNA-binding domain, the heme pocket appears to yield more conformational freedom for the ligand. This is in agreement with the observed slower kinetics of CO and NO recombination, as well as with the higher rate of displacement of the internal ligand, presumably a methionine residue (12). While obviously the ligand-binding properties of the heme domain influence the DNA-binding properties and transcriptional activity of the DNA-binding region, interestingly our results demonstrate that a reverse influence also occurs. One may speculate that the DNAbinding domain exerts a strain on the heme pocket that as a result becomes more open. Unfortunately structural data on RcoM proteins that could shine light on this matter on a molecular level are still missing. In general terms, the influence of DNA-binding on the CO recombination kinetics (as discussed below) supports the notion of a bidirectional effect between the two domains that might have a regulatory role.

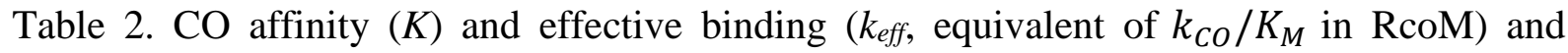
dissociation ( $k_{\text {off }}$ ) rates for full-length RcoM-2, the heme domain RcoMH-2 and other 6coordinate heme-based sensor proteins.

\begin{tabular}{|l|c|c|c|}
\hline & $k_{\text {eff }}\left(\mathrm{M}^{-1} \mathrm{~s}^{-1}\right)$ & $k_{\text {off }}\left(\mathrm{s}^{-1}\right)$ & $K\left(M^{-1}\right)$ \\
\hline RcoM-2 $^{a}$ & $16 \times 10^{3}$ & $64 \times 10^{-6}$ & $0.25 \times 10^{9 b}$ \\
& & & \\
RcoMH-2 $^{c}$ & $15 \times 10^{3}$ & $3.5 \times 10^{-6}$ & $4 \times 10^{9 b}$ \\
\hline EcDosH & $1.1 \times 10^{3 d} \quad$ & $0.011^{g}$ & $0.1 \times 10^{6 d}$ \\
& $7.8 \times 10^{3 e}$ & $0.078^{g}$ & \\
\hline CooA $^{h}$ & $8 \times 10^{3}$ & 0.02 & $0.45 \times 10^{6}$ \\
& $1.2 \times 10^{3}$ & 0.002 & \\
\hline NPAS2 $^{i}$ PASA & $370 \times 10^{3}$ & $\sim 0.5^{g}$ & $0.5-1 \times 10^{6}$ \\
\multicolumn{1}{|c|}{ PASB } & $40 \times 10^{3}$ & $0.8^{g}$ & $0.048 \times 10^{6}$ \\
\hline SUR2A $^{j}$ & $270 \times 10^{3}$ & 0.05 & $1.6 \times 10^{6}$ \\
\hline Neuroglobin (human) $^{k}$ & $150 \times 10^{3}$ & 0.014 & $11 \times 10^{6}$ \\
\hline
\end{tabular}

\footnotetext{
${ }^{a}$ This work

${ }^{b}$ Computed from $k_{\text {eff }}$ and $k_{\text {off }}$

${ }^{c}$ From ref. (12)

${ }^{d}$ From ref. (26)

${ }^{e}$ From ref. (27)
} 
$f$ using stopped flow; substantially higher $k_{\text {eff }}$ values were determined using flash photolysis (2830 ), see discussion in (28)

${ }^{g}$ Computed from $k_{\text {eff }}$ and $K$

${ }^{h}$ From ref. (31), biphasic kinetics

${ }^{i}$ From ref. (7), NPAS2 contains two heme-binding PAS domains

${ }^{j}$ From ref. (8)

${ }^{k}$ From ref. (32)

Whereas the measured CO dissociation rate of RcoM-2, $64 \times 10^{-6} \mathrm{~s}^{-1}$, is higher than that of RcoMH-2, it is still much lower than that of most other heme proteins, including other 6coordinate heme-based sensors (Table 2). Assuming the intrinsic thermal dissociation rate of the Fe-CO bond is similar in all systems, this difference is likely mostly due to the different yields in heme-CO geminate recombination. From the difference in dissociation rates we can estimate the CO escape probability in the RcoMH-2 heme-domain at $\sim 0.03 \%$, which is below our direct detection limit. Indeed, for most other 6-coordinate heme-based sensor proteins in Table 2 the geminate rebinding yield is much lower $(8,18,24,25,28,33,34)$ and the off-rate is 2-3 orders of magnitude higher than for RcoM-2. This is of the same order as the CO escape yield measured for RcoM-2 on the nanosecond time scale ( 0.5\%, Fig. 4). Furthermore the relatively slow CO dissociation in CooA (slowest phase $2 \times 10^{-3} \mathrm{~s}^{-1}$, (31)) correlates with a relatively low CO escape (a few percent $(18,25,33)$ ). Altogether, the CO off-rate is modulated by the escape probability from the heme pocket of dissociated $\mathrm{CO}$ and in RcoM proteins this modulation has led to extremely slow CO release from the protein.

In contrast to the dissociation rate, the effective $\mathrm{CO}$ binding rate (at low CO concentrations) is very similar in RcoM-2 and RcoMH-2 (and of the same order as in other heme proteins, Table 2). This finding is indicative of a similar rate of $\mathrm{CO}$ reaching the heme pocket, suggesting that in full-length RcoM-2 CO enters the protein directly at the heme domain and the intra-protein migration pathway does not pass via the DNA binding domain. Taking together the measured binding and dissociation rates, we determined the $K_{d}$ for CO at $4 \mathrm{nM}$ for RcoM-2 and 250 pM for RcoMH-2; these high affinities being largely determined by the 
properties of the heme pocket hindering CO escape. Even for the full-length protein these are among the highest CO affinities known. As discussed in our previous work (12) they are on the order of those observed for some truncated hemoglobins (35) that display slow ligand exchange, but where CO does not need to displace an internal ligand.

Our determination of a $K_{d}$ of $4 \mathrm{nM}$ for CO binding to RcoM-2 is in general agreement with the assessment of a value below $\sim 100 \mathrm{nM}$ by direct titration in the highly homologous RcoM-1 sensor (14). As has been discussed (14), these findings and the notion that aerobic RcoM sensors should be sensitive to persistent CO levels in the low nanomolar range, where CO oxidation in Burkholderia occurs (36), concord. The observed slow ligand exchange in this range also avoids rapid fluctuations in the sensor activity.

Fluorescence anisotropy-based DNA-binding assays were carried out to quantitatively determine the affinity of RcoM-2 to 5' Texas Red-labelled binding region (14) in its active CObound and inactive forms. In the FeIII form no DNA binding occurs, whereas for CO-bound RcoM-2, the observed anisotropy saturation upon protein addition indicates sequence-specific binding. The observed DNA binding constant $\mathrm{K}_{d}$ of $<2 \mathrm{nM}$ is higher than what has been observed under the same assay conditions for the NO-dependent transcriptional regulator DNR (44 nM (18) ) and the anaerobic CO-dependent transcriptional activator CooA (values between 6 and $16 \mathrm{nM}$ have been reported $(18,37,38))$. It is in agreement with a high functional affinity, similar to what has been observed for binding of RcoM-1 to the $a+b+c$ region (14), and an efficient CO-specific recruitment of RcoM-2.

Finally, our experiments show a small but significant effect of DNA substrate binding on the dynamics of CO recombination in the active site (Fig. 4), in general agreement with the bidirectional influence between the sensor and DNA-binding domains. Upon DNA binding the overall dynamics accelerate, a finding that is qualitatively similar to that observed in CooA (25). For RcoM-2, where the rebinding kinetics can be described with two exponentials of 
similar amplitude, DNA binding results in enhancement of the faster (170-ps) phase, which is also the dominant phase for the isolated heme domain. This comparison indicates that DNA binding induces a more rigid configuration of the heme domain with less configurational freedom for the dissociated CO. A similar conclusion was reached for CooA, based on narrowing of a wider range of rate distributions upon DNA binding (25) (a feature itself not occurring in RcoM-2). Altogether, these results suggest that this is a general feature for hemebased gas-regulated transcription factors.

In conclusion, we have demonstrated that the $\mathrm{CO}$ affinity in RcoM-2 is regulated by the ligand dynamics properties in the heme pocket, which are fine-tuned by long-range interactions with the DNA-binding domain. Whereas CO binding to the isolated heme domain is quasiirreversible, these interactions allow reversible CO binding in the full-length RcoM-2 protein. The resulting CO affinity ( $K_{d} 4 \mathrm{nM}$ ) is high compared to other CO-regulated systems, but brings the sensor in the sensitivity range expected for CO oxidation under aerobic conditions. Studies of the precise molecular origin of the important dynamic properties of the heme pocket must await the availability of structural data on the protein.

\section{ACCESSION CODE}

RcoM-2 Q13IY4

\section{ACKNOWLEDGMENT}

M.S. is supported by a municipal grant from Kafarhatta (Lebanon).

\section{SUPPORTING INFORMATION}

Table S1 with absorption maxima of different forms of RcoM-2 full length and isolated heme domain. 


\section{REFERENCES}

1. Shimizu, T., Huang, D., Yan, F., Stranava, M., Bartosova, M., Fojtikova, V., and Martinkova, M. (2015) Gaseous $\mathrm{O}_{2}$, NO, and CO in Signal Transduction: Structure and Function Relationships of Heme-Based Gas Sensors and Heme-Redox Sensors, Chem. Rev. 115, 6491-6533.

2. Négrerie, M. (2019) Iron transitions during activation of allosteric heme proteins in cell signaling, Metallomics 11, 868-893.

3. Boczkowski, J., Poderoso, J. J., and Motterlini, R. (2006) CO-metal interaction: vital signaling from a lethal gas, Trends Biochem. Sci. 31, 614-621.

4. Gullotta, F., di Masi, A., Coletta, M., and Ascenzi, P. (2012) CO metabolism, sensing, and signaling, Biofactors 38, 1-13.

5. Heinemann, S. H., Hoshi, T., Westerhausen, M., and Schiller, A. (2014) Carbon monoxide - physiology, detection and controlled release, Chem. Comm. 50, 3644-3660.

6. Kim, H., Ryter, S., and Choi, A. (2006) CO as a cellular signalling molecule, Annu. Rev. Pharmacol.Toxicol. 46, 411-449.

7. Dioum, E. M., Rutter, J., Tuckerman, J. R., Gonzalez, G., Gilles-Gonzalez, M.-A., and McKnight, S. L. (2002) NPAS2: A Gas-Responsive Transcription Factor, Science 298, 2385-2387.

8. Kapetanaki, S. M., Burton, M. J., Basran, J., Uragami, C., Moody, P. C. E., Mitcheson, J. S., Schmid, R., Davies, N. W., Dorlet, P., Vos, M. H., Storey, N. M., and Raven, E. (2018) A mechanism for CO regulation of ion channels, Nature Comm. 9, 907.

9. Ascenzi, P., Bocedi, A., Leoni, L., Visca, P., Zennaro, E., Milani, M., and Bolognesi, M. (2004) CO Sniffing through Heme-based Sensor Proteins, IUBMB Life 56, 309-315.

10. Lanzilotta, W. N., Schuller, D. J., Thorsteinsson, M. V., Kerby, R. L., Roberts, G. P., and Poulos, T. L. (2000) Structure of the CO sensing transcription activator CooA, Nat. Struct. Biol. 7, 876-880.

11. Kerby, R. L., Youn, H., and Roberts, G. P. (2008) RcoM: A New Single-Component Transcriptional Regulator of CO Metabolism in Bacteria, J. Bacteriol. 190, 3336-3343.

12. Bouzhir-Sima, L., Motterlini, R., Gross, J., Vos, M. H., and Liebl, U. (2016) Unusual Dynamics of Ligand Binding to the Heme Domain of the Bacterial CO Sensor Protein RcoM-2, J. Phys. Chem. B 120, 10686-10694.

13. Bowman, H. E., Dent, M. R., and Burstyn, J. N. (2016) Met104 is the CO-replaceable ligand at $\mathrm{Fe}(\mathrm{II})$ heme in the CO-sensing transcription factor BxRcoM-1, J. Biol. Inorg. Chem. 21, 559-569.

14. Kerby, R. L., and Roberts, G. P. (2012) Burkholderia xenovorans RcoMBx-1, a Transcriptional Regulator System for Sensing Low and Persistent Levels of Carbon Monoxide, J. Bacteriol. 194, 5803-5816.

15. Marvin, K. A., Kerby, R. L., Youn, H., Roberts, G. P., and Burstyn, J. N. (2008) The Transcription Regulator RcoM-2 from Burkholderia xenovorans Is a Cysteine-Ligated Hemoprotein That Undergoes a Redox-Mediated Ligand Switch, Biochemistry 47, 9016-9028.

16. Smith, A. T., Marvin, K. A., Freeman, K. M., Kerby, R. L., Roberts, G. P., and Burstyn, J. N. (2012) Identification of Cys94 as the distal ligand to the Fe(III) heme in the transcriptional regulator RcoM-2 from Burkholderia xenovorans, J. Biol. Inorg. Chem. 17, 1071-1082. 
17. Pinhancos, R. C., Bowman, H. E., Dent, M. R., Young, B. H., Berndsen, C. E., and Burstyn, J. N. (2017) The Heme-Binding PAS Domain Mediates Dimerization in the CO-Sensing Transcription Factor BxRcoM-1, FASEB J. 31, 908.15.

18. Lobato, L., Bouzhir-Sima, L., Yamashita, T., Wilson, M. T., Vos, M. H., and Liebl, U. (2014) Dynamics of the heme-binding bacterial gas sensing dissimilative nitrate respiration regulator (DNR) and activation barriers for ligand binding and escape, $J$. Biol. Chem. 289, 26514-26524.

19. Antonini, E., and Brunori, M. (1971) Hemoglobin and myoglobin in their reactions with ligands, North-Holland, Amsterdam.

20. Creze, C., Ligabue, A., Laurent, S., Lestini, R., Laptenok, S. P., Kuhn, J., Vos, M. H., Czjzek, M., Myllykallio, H., and Flament, D. (2012) Modulation of the Pyrococcus abyssi NucS endonuclease activity by the replication clamp PCNA at functional and structural levels, J. Biol. Chem. 287, 15648-15660.

21. Copeland, R. (2004) Enzymes: A Practical Introduction to Structure, Mechanism, and Data Analysis, John Wiley \& Sons.

22. Laptenok, S. P., Bouzhir-Sima, L., Lambry, J.-C., Myllykallio, H., Liebl, U., and Vos, M. H. (2013) Ultrafast real time visualization of the active site flexibility of the flavoenzyme thymidylate synthase ThyX, Proc. Natl. Acad. Sci. U.S.A. 110, 8924-8929.

23. Silkstone, G., Jasaitis, A., Wilson, M. T., and Vos, M. H. (2007) Ligand dynamics in an electron-transfer protein: picosecond geminate recombination of carbon monoxide to heme in mutant forms of cytochrome $c$, J. Biol. Chem. 282, 1638-1649.

24. Vos, M. H. (2008) Ultrafast dynamics of ligands within heme proteins, Biochim. Biophys. Acta 1777, 15-31.

25. Benabbas, A., Karunakaran, V., Youn, H., Poulos, T. L., and Champion, P. M. (2012) Effect of DNA Binding on Geminate CO Recombination Kinetics in CO-sensing Transcription Factor CooA, J. Biol. Chem. 287, 21729-21740.

26. Delgado-Nixon, V. M., Gonzalez, G., and Gilles-Gonzalez, M. A. (2000) Dos, a hemebinding PAS protein from Escherichia coli, is a direct oxygen sensor, Biochemistry 39, 2685-2691.

27. Taguchi, S., Matsui, T., Igarashi, J., Sasakura, Y., Araki, Y., Ito, O., Sugiyama, S., Sagami, I., and Shimizu, T. (2004) Binding of Oxygen and Carbon Monoxide to a Heme-regulated Phosphodiesterase from Escherichia coli: KINETICS AND INFRARED SPECTRA OF THE FULL-LENGTH WILD-TYPE ENZYME, ISOLATED PAS DOMAIN, AND MET-95 MUTANTS, J. Biol. Chem. 279, 33403347.

28. Liebl, U., Bouzhir-Sima, L., Kiger, L., Marden, M. C., Lambry, J.-C., Négrerie, M., and Vos, M. H. (2003) Ligand binding dynamics to the heme domain of the oxygen sensor Dos from Escherichia coli, Biochemistry 42, 6527-6535.

29. Ishitsuka, Y., Araki, Y., Tanaka, A., Igarashi, J., Ito, O., and Shimizu, T. (2008) Arg97 at the Heme-Distal Side of the Isolated Heme-Bound PAS Domain of a Heme-Based Oxygen Sensor from Escherichia coli (Ec DOS) Plays Critical Roles in Autoxidation and Binding to Gases, Particularly $\mathrm{O}_{2}$, Biochemistry 47, 8874-8884.

30. Lechauve, C., Bouzhir-Sima, L., Yamashita, T., Marden, M. C., Vos, M. H., Liebl, U., and Kiger, L. (2009) Heme Ligand Binding Properties and Intradimer Interactions in the Full-length Sensor Protein Dos from Escherichia coli and Its Isolated Heme Domain, J. Biol. Chem. 284, 36146-36159.

31. Puranik, M., Nielsen, S. B., Youn, H., Hvitved, A. N., Bourassa, J. L., Case, M. A., Tengroth, C., Balakrishnan, G., Thorsteinsson, M. V., Groves, J. T., McLendon, G. L., Roberts, G. P., Olson, J. S., and Spiro, T. G. (2004) Dynamics of Carbon Monoxide Binding to CooA, J. Biol. Chem. 279, 21096-21108. 
32. Dewilde, S., Kiger, L., Burmester, T., Hankeln, T., Baudin-Creuza, V., Aerts, T., Marden, M. C., Caubergs, R., and Moens, L. (2001) Biochemical Characterization and Ligand Binding Properties of Neuroglobin, a Novel Member of the Globin Family, $J$. Biol. Chem. 276, 38949-38955.

33. Kumazaki, S., Nakajima, H., Sakaguchi, T., Nakagawa, E., Shinahara, H., Yoshihara, K., and Aono, S. (2000) Dissociation and recombination between ligands and heme in a CO-sensing transcriptional activator CooA, J. Biol. Chem. 275, 38378-38383.

34. Abbruzzetti, S., Faggiano, S., Bruno, S., Spyrakis, F., Mozzarelli, A., Dewilde, S., Moens, L., and Viappiani, C. (2009) Ligand migration through the internal hydrophobic cavities in human neuroglobin, Proc. Natl. Acad. Sci. U.S.A. 106, 18984-18989.

35. Milani, M., Pesce, A., Nardini, M., Ouellet, H., Ouellet, Y., Dewilde, S., Bocedi, A., Ascenzi, P., Guertin, M., and Moens, L. (2005) Structural bases for heme binding and diatomic ligand recognition in truncated hemoglobins, J. Inorg. Biochem. 99, 97-109.

36. Weber, C. F., and King, G. M. (2012) The phylogenetic distribution and ecological role of carbon monoxide oxidation in the genus Burkholderia, FEMS Microbiol. Ecol. 79, 167-175.

37. Hines, J. P., Dent, M. R., Stevens, D. J., and Burstyn, J. N. (2018) Site-directed spin label electron paramagnetic resonance spectroscopy as a probe of conformational dynamics in the Fe(III) "locked-off" state of the CO-sensing transcription factor CooA, Protein Sci. 27, 1670-1679.

38. Thorsteinsson, M. V., Kerby, R. L., Conrad, M., Youn, H., Staples, C. R., Lanzilotta, W. N., Poulos, T. J., Serate, J., and Roberts, G. P. (2000) Characterization of Variants Altered at the N-terminal Proline, a Novel Heme-Axial Ligand in CooA, the CO-sensing Transcriptional Activator, J. Biol. Chem. 275, 39332-39338. 